Página inicial: 241 - Página final: 254

TIPO DE ARTÍCULO: de Investigación

\title{
FACTORES CAUSALES DE LA EXPLOTACIÓN SEXUAL INFANTIL EN NIÑOS, NIÑAS Y ADOLESCENTES EN COLOMBIA.
}

CAUSAL FACTORS OF CHILD SEXUAL EXPLOITATION IN BOYS, GIRLS, AND ADOLESCENTS IN COLOMBIA.

\begin{tabular}{|lll|}
\hline Recibido: Mayo 2014 & Revisado: Septiembre 2014 & Aceptado: Octubre 2014 \\
\hline
\end{tabular}

Por: Nora H. Londoño ${ }^{1}$, Diana Valencia ${ }^{2}$, Mario Garcia ${ }^{3}$, Catalina Restrepo. ${ }^{4}$

\section{RESUMEN:}

El propósito de la presente investigación fue identificar factores causales de la explotación sexual comercial en la infancia y la adolescencia en Colombia. Para ello se analizaron tres casos de estudio: Medellín, Sincelejo y Magangué. Se realizaron talleres y se aplicaron encuestas en cada una de las ciudades en Instituciones gubernamentales encargadas de intervenir el fenómeno y en algunas instituciones educativas, con la participación de funcionarios, profesionales, docentes, directivos y estudiantes. Para el análisis de datos se realizó una matriz explicativa con la participación de 4 jurados. Las categorías de análisis fueron factores medioambientales, familiares e individuales.

\section{PALABRAS CLAVE:}

Explotación sexual comercial infantil, educación, prevención, abuso sexual.

\section{ABSTRACT:}

The purpose of this piece of research aimed to identify causal factors of trade sexual exploitation on children and adolescents in Colombia. For this purpose, three case studies were analyzed: Medellin, Sincelejo, and Magangué. Workshops were conducted and surveys were implemented in each of the cities in both governmental institutions, in charge of intervening the phenomenon and in some educational institutions, with the participation of officials, professionals, teachers, directors, and students. As for the data analysis, an explanatory matrix was carried out, with the participation of 4 judges. The categories of analysis were those related to the environment, the family, and the individual.

\section{KEY WORDS:}

Child Trade Sexual Exploitation, Education, Prevention, and Sexual Abuse.

\footnotetext{
${ }^{1}$ PhD. Psicóloga. Docente investigadora Universidad de San Buenaventura, Medellín (Colombia). Contacto: doctorado.psicologia@usbmed.edu.co.

${ }^{2}$ Arquitecta, Mg., Candidata a Doctora en ciencias técnicas. Docente invetigadora Universidad de San Buenaventura, Medellin (Colombia). Contacto: Diana.Valencia@usbmed.edu.co.

${ }^{3}$ Abogado, Mg. Asociación ATUCSARA y docente Universidad de San Buenaventura, Medellín (Colombia).

${ }^{4}$ Psicóloga, Especialista, Universidad de San Buenaventura, Medellín (Colombia).
} 


\section{Introducción.}

La explotación sexual comercial es uno de los fenómenos nacionales e internacionales más complejos dado su carácter cambiante y diferencial dependiendo de los contextos donde se genera. La mayoría de los niños y adolescentes que practican esta actividad son obligados y no tienen la capacidad de comprender lo que realmente sucede o por qué suceden las cosas. Se calcula que más de un millón de niños, niñas y adolescentes son víctimas de explotación sexual comercial. Entidades como Esperanza para la familia, reporta cifras elevadas de niños, niñas y adolescentes explotadas sexualmente en diferentes países: se estima que 650.000 menores son explotados en las Filipinas, 400.000 en la India, 200.000 en Tailandia, 140.000 en Costa Rica, 25.000 en República Dominicana, 400.000 en Venezuela, 20.000 en Colombia y 10.000 en Chile. Por otro lado, la Organización Internacional para el Trabajo reporta en Chile al menos 3.719 niños, niñas y adolescentes víctimas, con edades entre los 10 y los 18 años, siendo el $80 \%$ mujeres y el $20 \%$ hombres (Bettio \& Nandi, 2010; OIT, 2007).

La explotación sexual comercial en la infancia y la adolescencia (ESCIA) puede definirse como aquella actividad lucrativa e ilicita que obedece a un conjunto de prácticas sociales propias de una cultura de ejercicio abusivo del poder y violencia frente a quienes, por su condición económica, o debido a sus sucesos de vida como la pobreza, suelen ser más vulnerables. Se trata de un fenómeno en donde el adulto concibe a la persona menor de edad como un objeto o producto comerciable, susceptible de ser comprado o vendido, para la satisfacción de sus propios deseos y fantasias (ICBF, UNICER, OIT, IPEC, Fundación Renacer, 2006). Más que prostitución infantil juvenil, abarca aspectos de la producción industrial pornográfica, el turismo sexual organizado, el matrimonio servil o forzado, trata de personas o tráfico de niños y adolescentes con fines de explotación que pueden ser sexual, laboral, mendicidad ajena, entre otros, sin importar el género, edad o lugar de origen de la posible víctima, y como hechos más recientes, el sexo y pornografia vía internet (Bonasso, 2003; Jakobsson \& Kotsadam, 2013; United Nations Office on Drugs and Crime, 2011).

Se afirma que la ESCIA, por implicar a menores, es en todo caso, una forma de explotación sexual inaceptable. Los menores tienen menos posibilidades de decidir libremente, con consentimiento informado, sobre los beneficios y costes de esta práctica sexual por dos razones bien evidentes: no tienen capacidad de dar consentimiento libre, siendo fácilmente manipulados y los efectos concomitantes y a largo plazo suelen ser muy negativos (Gutiérrez, Vega, \& Rodriguez, 2008).

Las propuestas de intervención deben estar encaminadas a proporcionar información sobre el fenómeno y sobre cómo responder apropiadamente ante los casos. El reconocimiento de los factores de riesgo frente a la victimización, además del reconocimiento sobre el comportamiento de las víctimas, posibilita la intervención a través de los servicios sociales especializados, dado que en general se detecta que los niños víctimas de ESCIA no reciben atención especializada ante su problemática y en los procedimientos se detectan grandes limitaciones (McMahonHoward \& Reimers, 2013).

La importancia de la prevención se plantea en los lineamientos legales de las políticas públicas de prevención, nacionales e internacionales, los cuales a través de principios éticos exponen sugerencias acerca de cómo proceder para que el hecho 
FACTORES CAUSALES DE LA EXPLOTACIÓN SEXUAL INFANTIL EN NIÑOS, NIÑAS Y ADOLESCENTES EN COLOMBIA.

no culmine y dar información sobre las penalidades para los establecimientos o personas que lo promuevan o permitan y para quienes obtienen "servicios sexuales" (Alexander, Meuwese, \& Wolthuis, 2000; OIT, 2011).

\section{$\underline{\text { Factores causales de explotación sexual comercial }}$}

Abordar este fenómeno desde la perspectiva multicausal implica el análisis de diferentes frentes que incluyen problemáticas de tipo económico, social, cultural y político. La desigualdad, la inequidad, la desintegración familiar, el consumismo, la migración rural-urbana, la discriminación, el abuso sexual intrafamiliar, el tráfico de drogas y de seres humanos pueden citarse como los principales factores de riesgo, los cuales se articulan como estructurales y estructurantes en la construcción de relaciones de violencia (Bonasso, 2003).

La explotación sexual comercial es un fenómeno multicausal, no se trata de un problema representativo de países en desarrollo; por el contrario es un fenómeno que se vive en todos los lugares del mundo, más frecuente en jóvenes que viven en la extrema pobreza, a menudo sin apoyo familiar importante y que se encuentran viviendo "la vida en las calles" como un joven sin hogar, fugitivos o tirado. Es en estas situaciones que la vulnerabilidad a la explotación sexual comercial aumenta. La recuperación es multifacética y complicada, y como tal debe incluir más de un proveedor de servicios (Williams, 2013).

El silencio. La mayor parte de niños y adolescentes de la calle que se prostituyen para sobrevivir ya fueron víctimas del silencio que envuelve la violencia sexual intrafamiliar. La sociedad igualmente adopta una postura de tolerancia, dimensionando el fenómeno como un problema privado y no como fenómeno social, contradictorio en el proceso de formación, civilización y evolución de la sociedad (Bonasso, 2003); se produce una falta de acción inmediata de la comunidad que por lo regular conoce la situación pero no hace nada en defensa del menor de edad (Díaz \& Rodríguez, 2006).

La pobreza. La pobreza y el hambre junto con el descuido o la complicidad de los familiares, han sido considerados factores de riesgo. La pobreza extrema afecta las condiciones de vida de las familias y favorecen que numerosos niños y adolescentes realicen trabajos en las calles, siendo una de las formas de trabajo infantil y de adolescentes que es considerado como factor de riesgo para la ESCIA. En este espacio, por sí mismo violento, las alternativas se cruzan con la delincuencia y la prostitución. Las niñas frecuentemente entran al mercado como empleadas domésticas, ambiente en el cual también son explotadas inclusive sexualmente (Camacho \& Trujillo, 2009; Díaz \& Rodríguez, 2006; Dutra-Thomé, CerqueiraSantos, \& Koller, 2011; López, 2004).

La estructura familiar. Se reporta en las investigaciones un mayor riesgo en las niñas cuando falta el padre y la madre trabaja o se dedica a la vida social en el barrio, descuidando a sus hijos. El explotador suele ser un familiar o vecino que se aprovecha de las carencias habituales de la niña y le ofrece, comúnmente, alimentos (Diaz \& Rodríguez, 2006). Se reflejan además una serie de relaciones violentas como el maltrato intrafamiliar, canales inadecuados de comunicación y falta de comprensión. Estas relaciones violentas y con deficiencia en el plano afectivo se convierten en un factor de vulnerabilidad que favorece la vinculación de estos niños, niñas y adolescentes a prácticas de explotación sexual comercial 
CAUSAL FACTORS OF CHILD SEXUAL EXPLOITATION IN BOYS, GIRLS, AND ADOLESCENTS IN COLOMBIA.

(Bettio \& Nandi, 2010). La interacción con familiares vinculados con prácticas de prostitución es considerada como un factor de vulnerabilidad hacia la situación de explotación sexual, debido a que la resistencia de los menores a vincularse a dichas prácticas puede verse disminuida al percibirlas como un estilo de vida (Camacho \& Trujillo, 2009).

Aspectos psicosociales. Otro factor analizado es la falta de autoridad del gobierno y de legislación que sancione o castigue estrictamente a las personas que ayudan a que crezcan de manera alarmante este tipo de actividades ilícitas. Según Garzón y Guerrero (2011), los factores que contribuyen a este fenómeno y a la trata de personas en Colombia son: la pobreza, la globalización, la migración, la prostitución, la cultura y el conflicto armado. La ausencia de políticas públicas de protección social y seguridad para los niños, niñas, adolescentes y sus familias facilitan las relaciones de mercado y de trabajo que operan mediante redes mundiales y cada vez aíslan de oportunidades y rehabilitación a las víctimas (Jakobsson \& Kotsadam, 2013). La influencia de las pandillas en sectores populares especialmente cuando la niña llega a la pubertad y es requerida sexualmente por miembros de estos grupos, los cuales pueden llegar a la coerción (Díaz \& Rodríguez, 2006).

Educación. Gran mayoría de niños y adolescentes explotados sexualmente están fuera del sistema regular de enseñanza o en situaciones de atraso escolar. La familia y la escuela son las dos principales instituciones sociales para proteger a la infancia. Por eso, cuando la familia y la escuela tienen un funcionamiento normalizado y los menores están integrados en estas instituciones (bien vinculados afectivamente en una familia que les cuida y protege e integradas en la escuela) es prácticamente imposible que ejerzan la prostitución. Si falla la integración del menor en estas instituciones, dejan de ser factores protectores y se convierten en factores de riesgo tales como ausencia de familia, la violencia intrafamiliar, la huida de casa, el absentismo escolar.

\section{Metodología.}

Muestra: se analizaron tres casos de estudio: Medellín, Sincelejo y Magangué. Las instituciones que participaron fueron: Instituto Colombiano de Bienestar Familiar ICBF, Policía de Infancia y Adolescencia, Proyecto Buen Vivir, Fiscalia, SIJIN, Medicina Legal, Secretaria de Bienestar Social, Secretaria de Educación, Corporación Amiga Joven, Corporación Primavera, Personeria, Crecer con Dignidad y Comisaria de Familia.

En el taller con docentes participaron 91 personas (30 Magangué, 8 en Sincelejo y 53 en Medellín), con los que se buscó describir el fenómeno y sensibilizar frente a la problemática. En el taller con jóvenes participaron 220 niños y adolescentes (50 Magangué, 25 Sincelejo y 145 en Medellin).

Procedimiento: se aplicaron encuestas para identificar los planes de intervención del fenómeno de la ESCIA en diferentes instituciones y se realizaron talleres en algunos colegios con directivos, docentes, y niños y adolescentes. Con éstos últimos, se elaboraron carteleras como instrumento facilitador para la descripción de los factores causales y de protección de la explotación sexual de acuerdo con la apreciación de ellos. 
Para el análisis de datos se realizó una matriz explicativa con la participación de 4 jurados, investigadores del proyecto identificados como Expertos (E1, E2, E3 y E4). Luego de haber reconocido los factores causales presentes en cada componente y clasificados por dimensión, se procedió a realizar un proceso de ponderación evaluando componentes y variables a través de la mirada de los Expertos. Este proceso de ponderación se realizó con la intención de determinar sobre que componentes y variables se deberian priorizar las estrategias de intervención.

\section{Resultados}

Los Expertos establecieron como principales factores causales de la ESCIA en los tres casos de estudio, Medellín, Magangué y Sincelejo, los relacionados con el medio ambiental, el familiar y el individual.

Cuadro 1.

\section{Factores relacionados con el medio}

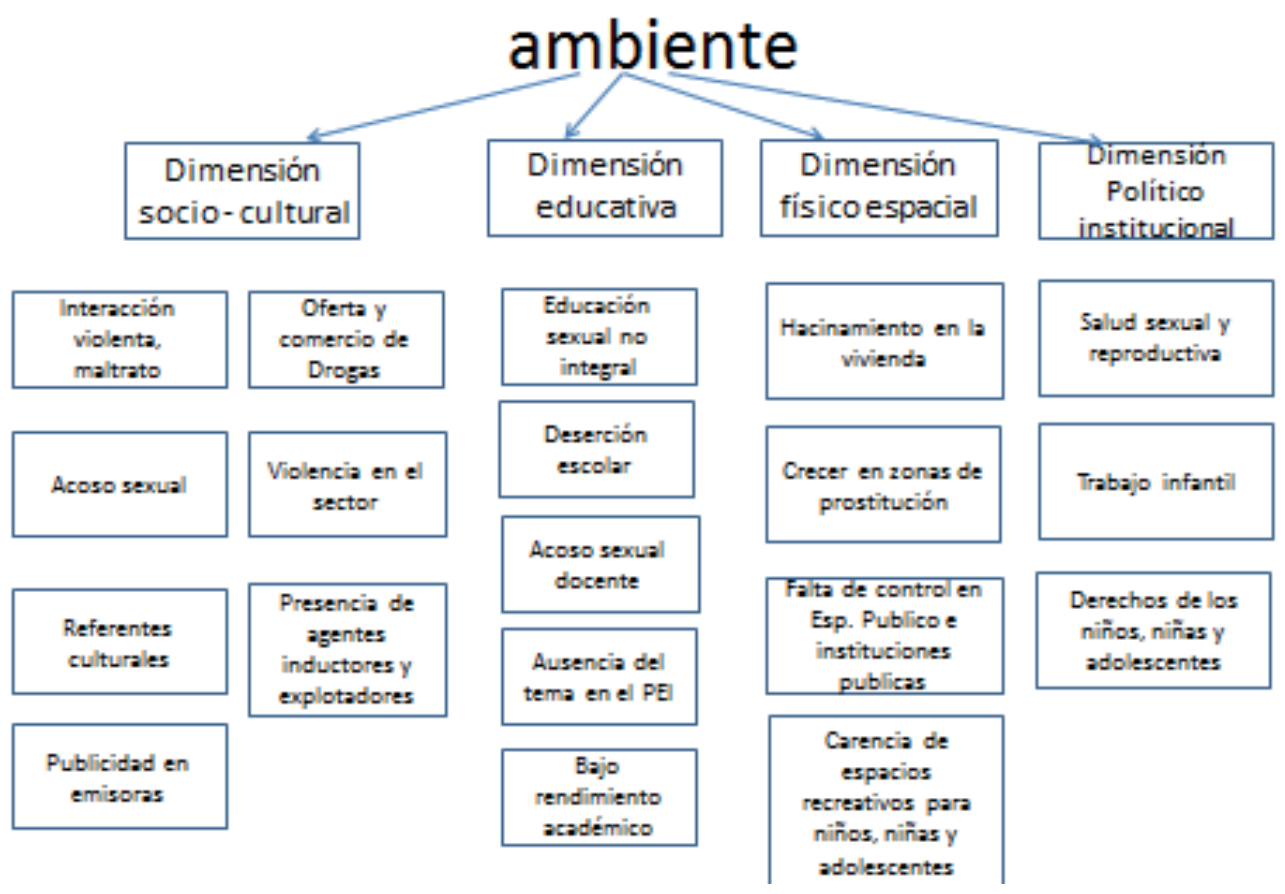

Fuente: propia 


\section{Cuadro 2.}

\section{Factores relacionados con el nivel familiar}
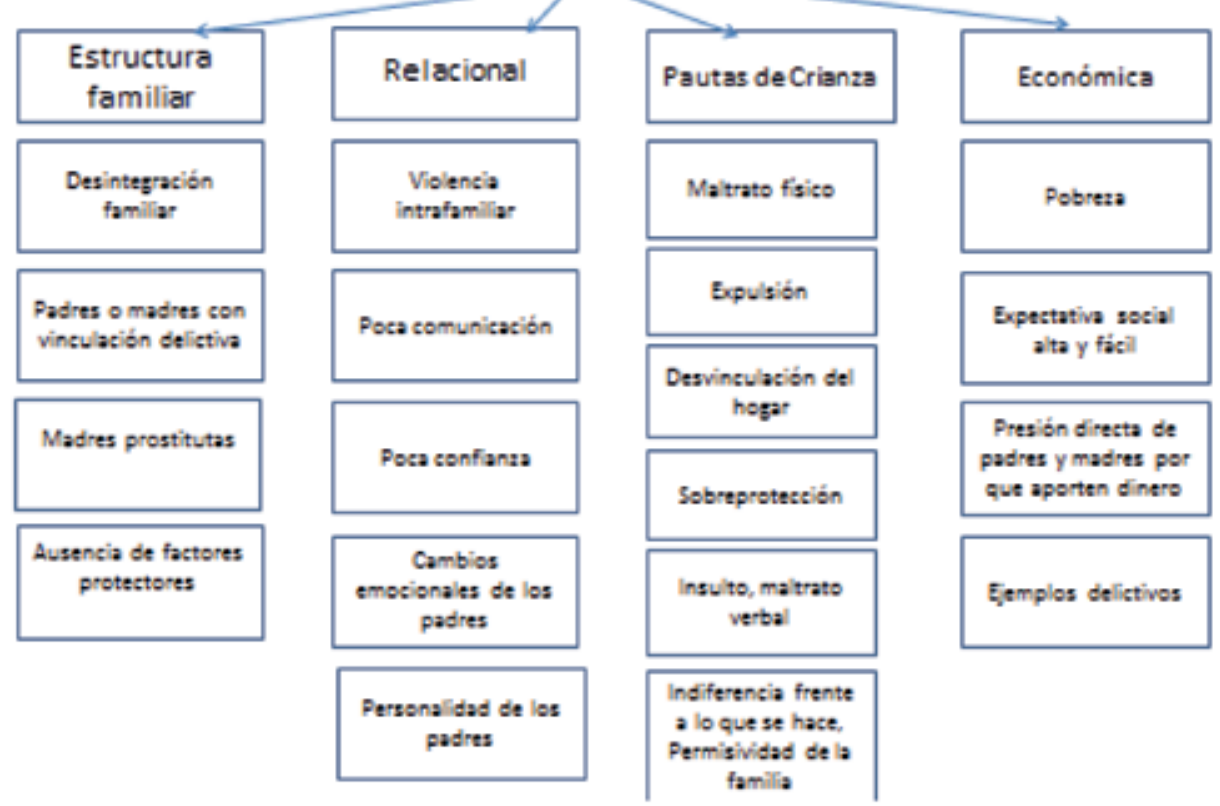

Expectativa socia alta y fácill

Presión directa de padres y madres por que aporten dinero

Fuente: propia

Cuadro 3.

Factores relacionados con el nivel

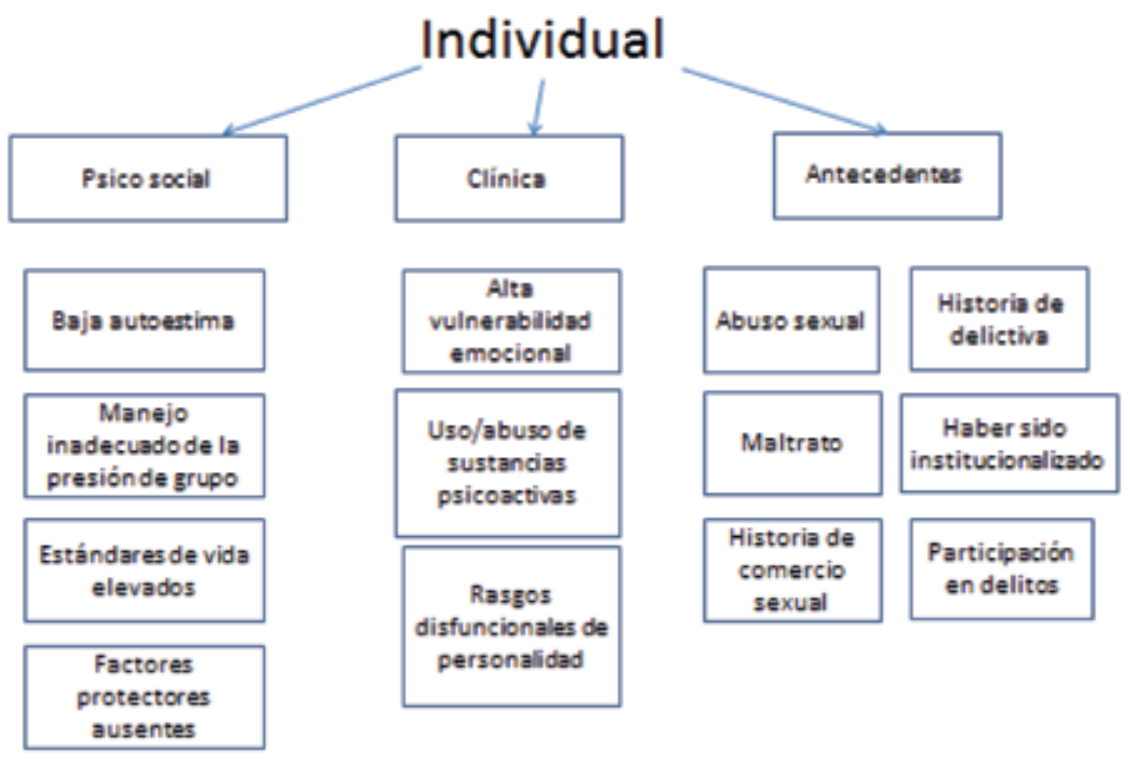

Fuente: propia 
La ponderación de factores de riesgo en la matriz causal por componentes generales mostró una mayor influencia de los factores de riesgo inherentes al Componente familiar (45\%), seguida por el Componente ambiental $(32.5 \%)$ y por el Individual (22.5\%) (Tabla 1).

Tabla 1. Ponderación factores de riesgo de la ESCIA por componentes generales

\begin{tabular}{lccccc}
\hline $\begin{array}{l}\text { Componentes } \\
\text { generales }\end{array}$ & \multicolumn{2}{l}{ Evaluadores expertos } & Erome \\
& E1 & E2 & E3 & E4 & dio \\
\hline Ambiental & 30 & 30 & 40 & 30 & $32.5 \%$ \\
Familiar & 45 & 55 & 30 & 50 & $45 \%$ \\
Individual & 25 & 15 & 30 & 20 & $22.5 \%$ \\
\hline
\end{tabular}

Fuente: propia

En el componente familiar, la dimensión Estructura familiar alcanzó los puntajes más elevados en la ponderación (35\%) (Tabla 2), siendo la variable Ejercicio de la prostitución de la madre el que mostró el peso más elevado (45\%) (Tabla 3).

Tabla 2. Ponderación factores de riesgo de la ESCIA en el componente familiar por dimensiones.

\begin{tabular}{|c|c|c|c|c|c|}
\hline \multirow{2}{*}{$\begin{array}{l}\text { Dimensiones } \\
\text { del componente } \\
\text { familiar }\end{array}$} & \multicolumn{4}{|c|}{ Evaluadores expertos } & \multirow{2}{*}{$\begin{array}{l}\text { Prome } \\
\text { dio }\end{array}$} \\
\hline & E1 & $\mathrm{E} 2$ & E3 & E4 & \\
\hline $\begin{array}{l}\text { Estructura } \\
\text { familiar }\end{array}$ & 30 & 30 & 45 & 35 & $35 \%$ \\
\hline Relacional & 15 & 20 & 10 & 20 & $16.3 \%$ \\
\hline $\begin{array}{l}\text { Pautas } \\
\text { crianza }\end{array}$ & 15 & 20 & 25 & 35 & $23.8 \%$ \\
\hline Económico & 49 & 30 & 20 & 10 & $27.3 \%$ \\
\hline
\end{tabular}

Fuente: propia. 
Tabla 3. Ponderación de las variables de riesgo de la ESCIA en el componente familiar, dimensión estructura familiar.

\begin{tabular}{llllll}
\hline $\begin{array}{l}\text { Variables de la } \\
\text { dimensión } \\
\text { estructura }\end{array}$ & E1 & E2 & E3 & E4 & $\begin{array}{l}\text { Pondera } \\
\text { ción }\end{array}$ \\
familiar
\end{tabular}

Fuente: Propia.

En el componente individual, la dimensión Antecedentes alcanzó un peso significativo (37\%) (Tablas 4), siendo la variable Abuso sexual la que alcanzó un mayor porcentaje de explicación (Tabla 5)

Tabla 4. Ponderación factores de riesgo de la ESCIA en el componente individual por dimensiones.

\begin{tabular}{lccccl}
\hline $\begin{array}{l}\text { Dimensiones } \\
\text { componente } \\
\text { individual }\end{array}$ & E1 & E2 & E3 & E4 & $\begin{array}{l}\text { Pondera } \\
\text { ción }\end{array}$ \\
\hline Psicosocial & 20 & 30 & 25 & 20 & $23.75 \%$ \\
Antecedentes & 45 & 40 & 50 & 50 & $46.25 \%$ \\
Clinica & 35 & 20 & 25 & 30 & $27.5 \%$ \\
\hline
\end{tabular}

Fuente:-propia. 
Tabla 5. Ponderación de las variables de riesgo de la ESCIA en el componente individual, dimensión Antecedentes.

\begin{tabular}{llcccl}
\hline $\begin{array}{l}\text { Variables de } \\
\text { antecedentes } \\
\text { del componente }\end{array}$ & E1 & E2 & E3 & E4 & $\begin{array}{l}\text { Pondera } \\
\text { ción }\end{array}$ \\
individual & & & & & \\
\hline $\begin{array}{l}\text { Abuso sexual } \\
\text { Maltrato }\end{array}$ & 30 & 30 & 25 & 35 & $30 \%$ \\
$\begin{array}{l}\text { Historia } \\
\text { comercio de } \\
\text { sexual }\end{array}$ & 30 & 20 & 30 & 15 & $23.8 \%$ \\
$\begin{array}{l}\text { Historia } \\
\text { delictiva }\end{array}$ & 10 & 30 & 25 & 20 & $21.3 \%$ \\
$\begin{array}{l}\text { Haber sido } \\
\text { institucionaliza } \\
\text { do }\end{array}$ & 10 & - & 10 & 15 & $8.8 \%$ \\
\hline & & & & & \\
\end{tabular}

Fuente: propia.

En la ponderación realizada al componente ambiental se determinó la dimensión Socio-cultural como la que más aportó a la matriz causal (Tabla 6), siendo la variable Presencia de agentes inductores el de mayor peso (22.5\%) (Tabla 7).

Tabla 6. Ponderación factores de riesgo de la ESCIA en el componente ambiental.

\begin{tabular}{lccccc}
\hline $\begin{array}{l}\text { Dimensiones } \\
\text { del componente } \\
\text { ambiental }\end{array}$ & $\begin{array}{l}\text { Evaluadores expertos } \\
\text { E1 }\end{array}$ & E2 & E3 & E4 & $\begin{array}{l}\text { Pondera } \\
\text { ción }\end{array}$ \\
\hline $\begin{array}{l}\text { Sociocultural } \\
\text { Fisico espacial }\end{array}$ & 20 & 20 & 15 & 15 & $17.5 \%$ \\
$\begin{array}{l}\text { Politico } \\
\text { institucional }\end{array}$ & 15 & 20 & 30 & 20 & $27.5 \%$ \\
Educativo & 15 & 30 & 35 & 30 & $27.5 \%$ \\
\hline
\end{tabular}

Fuente: propia. 
CAUSAL FACTORS OF CHILD SEXUAL EXPLOITATION IN BOYS, GIRLS, AND ADOLESCENTS IN COLOMBIA.

Tabla 7. Ponderación de las variables de riesgo de la ESCIA en el componente ambiental, dimensión Psicosocial.

\begin{tabular}{|c|c|c|c|c|c|}
\hline \multirow{2}{*}{$\begin{array}{l}\text { Variables de la } \\
\text { dimensión } \\
\text { sociocultural } \\
\text { del componente } \\
\text { ambiental }\end{array}$} & \multicolumn{5}{|c|}{ Evaluadores expertos } \\
\hline & E1 & E2 & E3 & E4 & $\begin{array}{l}\text { Pondera } \\
\text { ción }\end{array}$ \\
\hline Interacción & 5 & 20 & 20 & 20 & $16.3 \%$ \\
\hline Acoso sexual & 15 & 25 & 15 & 10 & $16.3 \%$ \\
\hline $\begin{array}{l}\text { Oferta comercio } \\
\text { de drogas }\end{array}$ & 10 & 15 & 10 & 20 & $13.8 \%$ \\
\hline $\begin{array}{l}\text { Violencia en el } \\
\text { sector }\end{array}$ & 25 & 20 & 10 & 10 & $16.3 \%$ \\
\hline $\begin{array}{l}\text { Presencia } \\
\text { agentes } \\
\text { inductores }\end{array}$ & 40 & 10 & 20 & 20 & $22.50 \%$ \\
\hline
\end{tabular}

Fuente: propia

\section{Discusión.}

El propósito de la presente investigación fue identificar factores causales de la explotación sexual comercial en niños, niñas y adolescentes en Colombia. Se analizaron tres casos de estudios, Medellín, Sincelejo y Magangué, y se estableció que el fenómeno de la explotación sexual comercial es percibido por la población en general como un fenómeno complejo y multicausal. La investigación logró identificar factores de riesgos comunes, en los cuales se centrará la discusión, y diferenciales pero con relación al peso de los factores de riesgo; en el caso de Medellín, especificamente, la problemática está más relacionada con la presión de grupo para lograr un mejor posicionamiento social, a diferencia de los casos Magangué y Sincelejo, donde la pobreza, asociada a las creencias culturales, ponen en riesgo al menor a participar en la red, presionados en muchos casos por las mismas familias o conocidos.

En los tres casos de estudio, se identificaron factores comunes causales agrupados en componentes y para cada uno de estos componentes dimensiones, y de éstas diferentes variables. Así, los tres componentes centrales identificados fueron el ambiental, el familiar y el individual. Para el ambiental se identificaron factores socioculturales, fisico-espaciales, politico-institucionales y educativos. Para el familiar se identificaron problemas de tipo relacionales, estructurales, de pautas de crianza y económicos. Y para el componente individual las dimensiones identificadas fueron los factores psicosociales, los antecedentes individuales e indicadores clinicos. Gran parte de estos componentes, dimensiones y variables fueron identificadas en el primer estudio realizado por los investigadores (Valencia 
et al., 2009) aunque se lograron precisar mayor número de variables identificadas como factores de riesgo.

Para la comprensión de este fenómeno de la explotación sexual comercial en menores de edad, las nociones predominantes tienen su asiento en el campo jurídico y se aproximan al problema desde un enfoque de derechos dado que es una violación fundamental de los derechos del niño; el niño es tratado como objeto sexual y mercancia, es esclavizado, lo separan de su familia, y lo exponen a graves peligros y enfermedades (Alexander, Meuwese \& Wolthuis, 2000; Declaración y Programa de Acción Primer Congreso Mundial contra la Explotación Sexual Comercial de Niños, 1996; Jakobsson \& Kotsadam, 2013; OIT, 2011; Unicef, 2004; Valencia et al, 2008).

Desde esta perspectiva, el menor se encuentra en una posición de desventaja y mayor vulnerabilidad en su condición social, jurídica y psicológica, y con gran riesgo de convertirse también en infractores de la ley (Condición Jurídica y Derechos del Niño, 2002), se relaciona con consumo de drogas y alcohol y con violencia explícita e implícita (ésta última por la concepción objetual) (Fernandez, 2011). De esta manera, antecedentes referentes a la historia personal son factores a tener en cuenta en el componente individual, los cuales hacen referencia al abuso sexual, historia delictiva, maltrato, haber sido institucionalizado y la historia de comercio sexual.

La estructuración familiar alcanzó un mayor nivel de riesgo cuando se encontraba desestructurada, la madre ejercía la prostitución y/o los padres se encontraban realizando actividades delictivas. Los factores psicosocial (analizados en el componente individual), se identificaron de mayor riesgo cuando se asociaban a problemas como baja autoestima, manejo inadecuado en la presión de grupos y estándares de vida elevados cuando presentan baja posición económica. Estos factores también fueron considerados relevantes en el estudio de Adamczyk (2012), facilitando la conexión con las ofertas de los proxenetas. La prostitución en los jóvenes es significativamente influenciada por sus amigos quienes dan una imagen de ayudarles a salir de los problemas.

En general se establece como derechos fundamentales de los niños tener una familia y no ser separados de ella, el cuidado y el amor (Unicef, 2006), de tal manera que los ambientes familiares sean estables y con un buen clima afectivo para proporcionan en sus miembros seguridad y afecto, elementos indispensables para un buen funcionamiento psicológico, tanto social como personal. Los niños, niñas y adolescentes no solo tienen el derecho a la vida, sino también a la calidad de la misma y a un ambiente sano en condiciones de dignidad y goce de todos sus derechos en forma prevalente (Unicef, 2004), siendo la calidad de vida esencial para su desarrollo integral (Salazar, 2007).

Por el contrario, un grado elevado de conflictos entre los padres, el abandono o la muerte de sus padres o familiares encargados, predisponen a la explotación sexual de NNA (ICBF et al., 2006). Estas condiciones incrementan en sentimientos de tristeza, malestar e infelicidad. La separación de los padres supone una de las experiencias más estresantes y dramáticas a los que puede enfrentarse los menores. Les genera inseguridad e incertidumbre con hondas repercusiones en edad adulta (Esperón \& Sanz, 2010).

Otra aproximación a este fenómeno debería lograrse desde el saber de la salud mental. Sin embargo, la clasificación diagnóstica no lo identifica como tal, sino 
dentro de los problemas relacionados con el maltrato infantil y negligencia, a manera de Abuso sexual, al describir conductas en las que se presiona al menor a participar en actos sexuales gratificantes para otros (American Psychiatric Association, 2013). Algunos autores diferencias abuso y explotación sexual, ambos dentro de la clasificación de violencia sexual, donde el intercambio económico o material se da en la explotación sexual (Dutra-Thomé, Cerqueira-Santos, \& Koller, 2011). En la presente investigación, la matriz explicativa identificó el fenómeno como una problemática que debe tratarse de manera compleja, con modelos multicausales que aborden lo jurídico, lo familiar y lo individual, y dentro de éstos lo referente a la salud mental.

Los indicadores clinicos, identificados como consecuencias emocionales del fenómeno en el menor de edad, se representan en vulnerabilidad emocional, abuso de sustancias psicoactivas y rasgos problemáticos de personalidad como conducta antisocial, impulsividad, y bajo control emocional. Los antecedentes como abuso sexual y haber participado en el comercio sexual también fueron representativos en el componente individual. Carson, Foster y Tripathi (2013) exponen los traumas psicológicos y emocionales asociados con el abuso sexual en niños y adolescentes y la necesidad de asistirles terapéuticamente.

Estudios como el de Bettio y Nandi (2010) enfatizaron de manera similar el abuso sexual como un antecedente importante en las mujeres explotadas sexualmente, y específicamente en América Latina cuando las familias son quienes las inducen (United Nations Office on Drugs and Crime, 2011). El menor es utilizado como un objeto sexual, como mercancía para el beneficio económico de terceras personas que se lucran gracias a sus necesidades.

La estructura socio cultural es la dimensión en la cual el individuo conforma las creencias de cada cultura y las jerarquiza en el estilo utilizado en las relaciones. Adicional a éste, las dimensiones referentes al espacio físico de la vivienda como el hacinamiento, espacios públicos, tolerancia al consumo y comercialización de droga, la prostitución y limitaciones recreativas, afectan la calidad de vida de NNA y ponen en riesgo a los menores al naturalizarse las actividades delictivas y el comercio sexual como maneras para disponer de recursos económicos y garantizar la supervivencia y la adaptación al medio social donde vive.

En conclusión, el fenómeno de la ESCIA es complejo y desde los diferentes factores causales se requieren propuestas de intervención pero contando como base los derechos fundamentales de los niños y la salud integral del menor. La ponderación de las categorías reflejó un mayor peso explicativo a los factores familiares, en especial los problemas relacionados con la estructura familiar, seguido por los factores individuales, en especial los antecedentes individuales como el abuso sexual, y por último los factores ambientales, en especial crecer en zonas de prostitución.

Como limitación del estudio, se considera que al ser la problemática demasiado global, el estudio no alcanzó identificar condiciones políticas internacionales que sí se lograria evidenciar al analizar casos de diferentes países los cuales son referidos en diversas investigaciones y que permiten diferenciar los casos en Latinoamérica como fueron los estudiados, con otros continentes y países. 


\section{Referencias bibliográficas.}

Adamczyk, A. (2012). Dank: The Commercial Sexual Exploitation of Children.

LFB Scholarly Publishing LLC, E1 Paso, Texas 2011. J Youth Adolescence, 41:12531255.

Alexander, S., Meuwese, S., \& Wolthuis, A. (2000). Policies and developments relating to the sexual exploitation of children: the legacy of the Stockholm conference. European Journal on Criminal Policy and Research, 8,475-501.

American Psychiatric Association (2013). Diagnostic and statistical manual of mental disorders. Fifth edition. DSM-5. Washington, D.C.: American Psychiatric Publishing.

Bettio, F. \& Nandi, T.K. (2010). Evidence on women trafficked for sexual exploitation: A rights based analysis. Eur J Law Econ, 29, 15-42.

Bonasso, A. (2003). La explotación sexual de niños, niñas y adolescentes en América Latina. Segunda Edición. Instituto Interamericano del Niño. Organización especializada de la OEA.

Carson, D.K., Foster, J.M., \& Tripathi, N. (2013). Child sexual abuse in India: Current Issues and Research. Psychol Stud, DOI, 10.1007/s 12646-013- 0198-6.

Camacho, L.B. \& Trujillo, M.A. (2009). La explotación sexual comercial infantil: una ganancia subjetiva. Rev.latinoam.cienc.soc.niñez juv 7(2), 1009-1025.

Condición Jurídica y Derechos del Niño (2002). Corte interamericana de Derechos Humanos, 28 de Agosto de 2002.

Díaz, O.S. \& Rodríguez, R. (2006). La explotación sexual de los niños en Cartagena de Indias y Bogotá, Colombia. Revista Infancia Adolescencia y Familia, 1(2), 247-258.

Dutra-Thomé, L., Cerqueira-Santos, E. \& Koller, S. H. (2011). Exploração sexual e trabalho na adolescência: Um estudo de caso. Universitas Psychologica, 10 (3), 881-896.

Esperón, C.S. \& Sanz, M.J.M. (2010). Manual de psiquiatría del niño y del adolescente. Madrid, España: Editorial Médica Panamericana.

Fernandez, M.C. (2011). El comercio sexual en Chile: ambigüedades y contradicciones discursivas. Anagramas rumbos sentidos comun., 9, (18), 71-81.

Garzón, L. \& Guerrero, C. (2011). Matrimonio servil: esclavas del sexo, un mundo que no te esperas conocer. Recuperado el 25 de septiembre de 2011, en: http:/ / www.slideshare.net/Lua_2401/matrimonio-servil-presentacin

Gutiérrez, R., Vega, L., \& Rodríguez, E. (2008). Problemas y dilemas éticos en la investigación de la explotación sexual comercial de niñas y niños (Spanish). Salud Mental, 31(5), 403-408. 
CAUSAL FACTORS OF CHILD SEXUAL EXPLOITATION IN BOYS, GIRLS, AND ADOLESCENTS IN COLOMBIA.

ICBF, UNICER, OIT, IPEC, FUNDACIÓN RENACER (2006). Plan de Acción Nacional para la prevención y erradicación de la Explotación Sexual Comercial de Niños, Niñas y Adolescentes menores de 18 años (ESCNNA). Bogotá, Colombia: ICBF y UNICEF.

Jakobsson, N. \& Kotsadam, A. (2013). The law and economics of international sex slavery: prostitution laws and trafficking for sexual exploitation. Eur J Law Econ, 35, 87-107.

López, J.M. (2004). La explotación sexual en adolescentes en Medellín. Medellín, Colombia. Secretaría de Bienestar Social y Centro de Investigación Sociales y Humanas CISH Universidad de Antioquia.

McMahon-Howard, J. \& Reimers, B. (2013). An evaluation of a child welfare training program on the commercial sexual exploitation of children (CSEC). Evaluation and Program Planning, 40, 1-9.

OIM (2011). Organización internacional para las migraciones. Conceptos generales de la trata de personas. Recuperado el 25 de septiembre de 2011, en:

http: / / www.oim.org.co/Programas / ContralaTratadePersonas / Conceptosgeneralesdetratadepersonas/tabid/179/language/es-CO/Default.aspx

Oficina Internacional del Trabajo OIT (2011). Con los ojos abiertos: cartilla para los maestros sobre el trabajo infantil. Recuperado el 25 de septiembre de 2011, en: http://white.oit.org.pe/ipec/documentos/guia_maestros_ti_col.pdf

OIT/IPEC (2007). Guía para prestadores de servicios de turismo. Protegiendo los derechos de niños, niñas y adolescentes. Prevención de la explotación sexual comercial de niños, niñas y adolescentes.

Salazar, E. (2007). Código de la infancia y la adolescencia. Bogotá: Leyer.

Unicef (2006). Convención sobre los derechos de los niños y las niñas. Fondo de las Naciones Unidas para la Infancia. Colombia y Venezuela.

Unicef (2004). Ciudades para la niñez. Bogotá: Beta Impresores Ltda.

United Nations Office on Drugs and Crime (2011). Trafficking in persons to Europe for sexual exploitation. Trends Organ Crim, 14, 224-234.

Valencia, D. E, Botero, C. A., Londoño, N.H., Palacios, Y., Valencia, P.A., Arias, B.E., Torres, J.J. \& Burgos, F. (2007). Factores causantes de explotación sexual comercial en niños, niñas y adolescentes en la costa Atlántica, municipios de Sincelejo y Barranquilla, con especial énfasis en población desplazada por la violencia. Universidad de San Buenaventura, Medellin (Colombia).

Williams, L. (2013). Adolescence and Commercial Sexual Exploitation: Prostituted Girls in the US, pp 449-468. In: D. S. Bromberg and W.T. O'Donohue. Handbook of Chilod and Adolescent Sexuality. Developmental and Forensic Psychology. Chapter 18 -. University of Massachusetts Lowell, Lowell, Massachusetts, Academic Press. 